\title{
Management and Microbiology of Perforated Appendicitis in Pediatric Patients: A 5-Year Retrospective Study
}

\author{
Alex S. Plattner · Jason G. Newland · Michael J. Wallendorf • \\ Baddr A. Shakhsheer
}

Received: June 1, 2021 / Accepted: July 12, 2021 / Published online: July 21, 2021

(C) The Author(s) 2021

\section{ABSTRACT}

Introduction: This study aims to assess the current epidemiology and microbiology of perforated appendicitis, how antibiotic choice and duration correlate with meaningful clinical outcomes, and whether serial white blood cell (WBC) counts provide clinical value.

Methods: Five-year retrospective cohort study, 2015-2019, among 333 consecutive children, ages 0-18 years, treated at St. Louis Children's Hospital for perforated appendicitis. Main outcomes included length of stay (LOS), postoperative abscess formation, and readmission. Statistical analysis was performed with uni- and multi-variate analyses.

Results: Intra-abdominal cultures most commonly grew Bacteroides fragilis (52\%) and Escherichia coli (50\%). Patients who initially

A. S. Plattner $\cdot$ J. G. Newland

Division of Infectious Diseases, Department of Pediatrics, Washington University in St Louis School of Medicine, St Louis, MO, USA

\section{J. Wallendorf}

Division of Biostatistics, Washington University in

St Louis School of Medicine, St Louis, MO, USA

B. A. Shakhsheer $(\bowtie)$

Division of Pediatric Surgery, Department of Surgery, Washington University in St Louis School of Medicine, 1 Children's Place, Suite 6110-CB 8235, St Louis, MO 63110, USA

e-mail: baddr@wustl.edu received broad-spectrum antibiotics (meropenem, piperacillin-tazobactam, fourth-generation cephalosporins) for perforated appendicitis had greater rates of postoperative abscess formation $(25 \%$ vs. $12 \%, p<0.01)$ and LOS (7.0 vs. 5.7 days, $p<0.01)$. Similarly, antibiotics at time of discharge were associated with greater postoperative abscess formation ( $22 \%$ vs. $9 \%, p<0.01)$ and LOS (6.4 vs. 5.6 days, $p=0.02$ ). However, discharge with strictly oral antibiotics was not correlated with greater LOS, postoperative abscess formation, or readmission rates compared to discharge without antibiotics. Serial WBC counts had no predictive value for LOS, postoperative abscess formation, or readmission.

Conclusions: Bacteroides fragilis and E. coli were the most common intra-abdominal microbes for perforated appendicitis among our cohort. In non-critically ill children, the routine use of broad-spectrum antibiotics or continuation of antibiotics beyond discharge was not correlated with improved clinical outcomes. Additionally, WBC counts were not correlated with meaningful clinical outcomes.

Keywords: Antibiotic stewardship; Complicated appendicitis; Pediatric surgery 


\section{Key Summary Points}

\section{Why carry out this study?}

Appendicitis is among the most common surgical emergencies, and perforated appendicitis continues to be a source of morbidity with significant practice variation in management of these children

Our article uses an extensive 5-year retrospective experience to address common management questions: the utility of the white blood cell (WBC) count, use of broad- versus narrowspectrum antibiotics, and practice of discharging patients home with antibiotics

\section{What was learned from the study?}

These data are important to show the WBC count, used to treat perforated appendicitis, is not reliable and lend further credence to the principal of treating the patient rather than the laboratory parameters

Furthermore, our article demonstrates that patients are over-treated with broad and lengthy antibiotic duration regimens with no patient benefit. Taken together, these data have important implications for a common pediatric problem

\section{INTRODUCTION}

Complicated appendicitis is one of the most common surgical emergencies in pediatrics, with the standard of care resting on the pillars of surgical resection and antibiotic treatment $[1,2]$. While the surgical aspect is relatively well defined, laparoscopic or open appendectomy, the roles of bacterial cultures and antibiotics, including agent, duration, and route, are less clear. Review of the current literature

demonstrates discord over the utility of intraoperative cultures in guiding antibiotic decision making and preventing subsequent intraabdominal abscesses, the most common postoperative complication of perforated appendicitis $[3,4]$. While the choice of antibiotics varies by surgeon and across institutions, the overall duration and coverage spectrum for perforated appendicitis has become shorter and more specific. Previous studies have shown antibiotic therapy consisting of metronidazole and a third-generation cephalosporin to be effective in the management for perforated appendicitis, although "broad-spectrum" antibiotics continue to be routinely used $[5,6]$. Following surgery, postoperative complications are potentially worse among patients discharged home with intravenous antibiotics compared to oral antibiotics [7-9]. Additionally, the approach of discharging patients without home oral antibiotic courses has been validated in the setting of a normal white blood cell (WBC) count [10-13].

WBC trends are routinely used as a diagnostic aide in both the diagnosis and subsequent management of perforated appendicitis. Although an initial WBC may guide the diagnosis or exclusion of appendicitis, the predictive value and subsequent monitoring of serial WBCs remain questionable. One study of 44 pediatric patients with perforated appendicitis showed no significant difference in admission WBC among patients who later developed postoperative abscesses versus those who did not [14]. Furthermore, no patient developed an abscess if they were tolerating a regular diet by postoperative day 3 . To this end, recent data suggest patients can be safely discharged home when clinically well (afebrile, tolerating a diet without nausea or emesis, pain controlled) without the need to check the WBC [15].

The objectives of this study were to: (1) describe the bacterial growth patterns observed in children with perforated appendicitis in a single large academic center; (2) determine whether there are correlations between antibiotic choice or duration and clinical outcomes in perforated appendicitis; (3) assess whether monitoring WBC correlates with meaningful clinical outcomes. 


\section{METHODS}

\section{Design, Setting, Participants}

This study received approval from the Washington University in St. Louis institutional review board (IRB), ID \#201908150. A retrospective cohort study was conducted among children, ages $0-18$ years, treated at St. Louis Children's Hospital (SLCH) for perforated appendicitis from 2015 to 2019. All cases admitted for appendectomy at SLCH during the study time frame were reviewed, and patients were included if the primary surgeon identified the appendix as being "perforated" in their operative findings at the time of removal $(N=335)$. Two patients were excluded. One patient had been admitted for several days at another hospital before transferring at the time of surgery, and records were limited. The other excluded patient had extensive comorbidities/ underlying pathology that confounded their presentation and subsequent hospital course. After exclusions, 333 consecutive pediatric patients remained for consideration.

\section{Exposures}

Initial management type was at the discretion of the attending surgeon. Patients who had surgery as the initial management underwent open or laparoscopic appendectomy within $24 \mathrm{~h}$ of admission. In some situations, a preoperative drainage procedure was performed by the interventional radiology service, also within $24 \mathrm{~h}$ of admission. In general, these were patients who presented with prolonged symptoms ( $>5$ days of pain) as well as imaging demonstrating a mature abscess (rim-enhancing, drainable by interventional radiology). Medical therapy was defined as antibiotics only without intent for immediate intervention, either surgical or radiologic. In both of these latter scenarios, patients returned weeks later for interval appendectomy and rarely required overnight admission. Perforated appendicitis was defined as presence of intra-luminal contents outside of the bowel at time of surgery (as per the operative note) [16].
Three distinct types of intra-abdominal cultures were identified based on time of collection. Intraoperative cultures were collected at the time of appendectomy. Preoperative drainage cultures were obtained from interventional radiology drain collection prior to interval appendectomy. Postoperative abscess cultures were obtained from interventional radiology drain following identification of a postoperative intra-abdominal abscess, usually $\geq 5$ days after initial appendectomy.

The initial antibiotic choice was defined as the preoperative antibiotics chosen by the surgical and medical teams at time of diagnosis. Broad-spectrum antibiotic coverage was defined as treatment with meropenem, piperacillin-tazobactam, and/or a fourth-generation cephalosporin. Narrow antibiotic coverage groups were exclusively treated with metronidazole plus a third-generation cephalosporin, most commonly ceftriaxone.

Admission and discharge WBC counts were evaluated, when available, for the patients in the study cohort. An admission WBC count $(N=316)$ was obtained either at a preceding institution or on arrival prior to the initial intervention, defined above. A discharge WBC count $(N=301)$ was only included in evaluation if it was obtained within $24 \mathrm{~h}$ prior to discharge.

\section{Main Outcomes and Measures}

Main outcome measures included length of stay (LOS) in days (determined by the number of midnights admitted), readmission within 30 days, postoperative abscess formation as defined by fluid collection requiring drainage as decided by the attending surgeon, and total days of antibiotic use.

\section{Statistical Analysis}

Univariable analysis was first performed using Fisher's exact tests and chi-square analyses for binary outcomes. A two-sample $t$-test was used for continuous variables. A multivariable logistic regression was subsequently conducted to assess the impact of multiple exposures (age, 
sex, race, admission $\mathrm{WBC}$, and initial broadspectrum antibiotic use) on postoperative abscess formation. The exposures of interest were chosen by the research team prior to performing the analysis. A $p$ value $<0.05$ was considered significant.

\section{RESULTS}

Of the 333 patients included in this study, 44\% were female, $78 \%$ were Caucasian, and $16 \%$ were African American. Female patients had a shorter average LOS (5.5 vs. 6.4 days, $p<0.01$ ) and shorter average antibiotic duration (9.7 vs. 11.2 days, $p=0.04$ ), but no significant differences were present in postoperative abscess formation or readmission rate. African American patients had no significant difference in age, antibiotic duration, postoperative abscess formation, or readmission rate compared to Caucasian patients. However, African American patients did have a longer average LOS (7.0 vs. 5.7 days, $p=0.02$ ). The mean age was 10.3 (SD 3.9) years (Table 1). The age group with the highest rate of postoperative abscesses was in children $<3$ years old (Fig. 1 ).

The majority of patients, $89 \%(N=295)$, with perforated appendicitis underwent a primary surgical intervention. The remaining patients were initially managed with either radiologic drainage $(N=24)$ or medical management $(N=14)$. The average patient in the surgical group was 2 years younger than those

Table 1 Study population demographics

\begin{tabular}{ll}
\hline & $\begin{array}{c}\text { Total population } \\
\text { demographics }\end{array}$ \\
\hline Average (standard deviation) & $10.3(3.9)$ \\
Age (years) & $6.0(3.0)$ \\
Total length of stay (days) & $10.5(6.4)$ \\
Antibiotic duration (days) & \\
Total (\%) & $52(16 \%)$ \\
Postoperative abscess & $23(7 \%)$ \\
Readmission & \\
\hline
\end{tabular}

in the preoperative drainage or medical management groups (10.1 vs. 12.1 vs. 12.8). The average total course of antibiotics was shortest in the surgical group (10.4 vs. 15.3 vs. 17.6$)$ but the readmission rate was approximately the same among all groups (7-8\%).

Preoperative drainage cultures were the most likely to show bacterial growth, with identifiable growth on $96 \%(25 / 26)$ of cultures, followed by $87 \%$ (40/46) growth on intraoperative cultures and $51 \%(25 / 49)$ growth on postoperative abscess cultures (Table 2). Bacteroides fragilis and Escherichia coli were the most prevalent bacteria isolated, occurring in 52\% and 50\%, respectively, of intra-abdominal cultures. Pseudomonas aeruginosa grew in $12 \%$ of cultures. Blood cultures had a positive growth rate of only 5\% (3/60). In 11 cases, a patient had both an intraoperative surgical site culture and a postoperative abscess culture (Table 3). Among these 11 cases, 2 had identical culture profiles, both growing $E$. coli and $P$. aeruginosa, with one case also growing Viridans group streptococcus on both cultures. In seven cases, intraoperative cultures had positive bacterial growth but the postoperative abscess culture was sterile. In only one case was an additional pathogen identified on the postoperative abscess that was not present in the intraoperative culture; the patient grew $B$. fragilis and $E$. coli on intraoperative culture before growing $E$. coli and $P$. aeruginosa on postoperative abscess culture.

Patients who were treated initially with broad-spectrum antibiotics prior to surgery had significantly higher rates of postoperative abscess formation $(25 \%$ vs. $12 \%, p<0.01)$, longer average LOS (7.0 vs. $5.7, p<0.01)$, and longer average antibiotic duration (13.5 vs. $10.4, p<0.01$ ), as seen in Table 4 . There was no significant difference in rate of readmission $(8 \%$ vs. $6 \%, p=0.61)$. Among patients with an initial surgical management $(N=295)$, outcomes were compared between patients that were discharged home with antibiotics (PO, IV, or a combination) $(N=180)$ versus patients whose antibiotics were discontinued at time of discharge $(N=115)$. Patients discharged with home antibiotics had a significantly higher rate of postoperative abscess formation (22\% vs. $9 \%$, $p<0.01)$ and longer average total LOS (6.4 vs. 


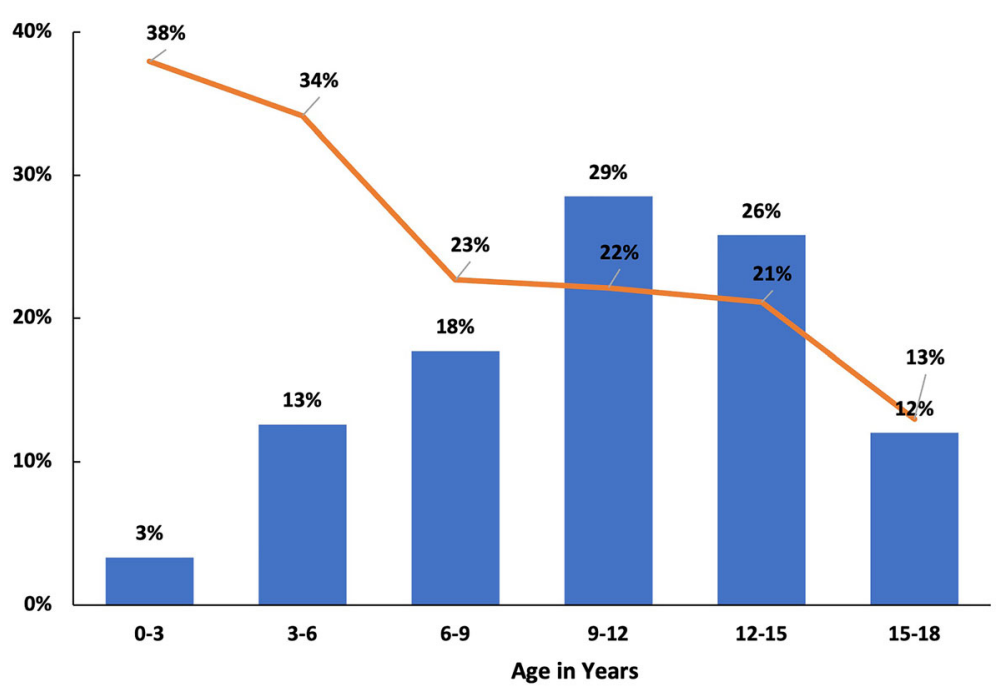

Fig. 1 Perforated appendicitis rates by age. The bar graph shows the study population segmented by age. The line graph represents perforation rate, by age, of all patients with appendicitis seen at St. Louis Children's Hospital from 2015-2019

5.6, $p=0.02)$. No significant differences in readmission rates were observed. In an attempt to account for variability in acuity, a comparison between patients discharged with only oral antibiotics $(N=135)$ versus patients discharged with no antibiotics $(N=115)$ was conducted. This analysis observed no difference in the rate of postoperative abscess formation ( $9 \%$ vs. $10 \%$, $p=0.79)$, readmission rate $(8 \%$ vs. $6 \%$, $p=0.39$ ), or average total LOS (5.7 vs. 5.6 days, $p=0.13)$.

The average admission WBC count among patients who developed postoperative abscess formation vs. those who did not was 17.5 vs. $17.0(p=0.60)$, respectively. Admission WBC count, regardless of age, was not correlated with subsequent LOS, antibiotic duration, postoperative abscess formation, or readmission, as outlined in Tables 5 and 6 . There was, however, a significantly higher average discharge WBC count among patients who were subsequently readmitted versus those without readmission (11.4 vs. 9.9, $p<0.01$ ). Additionally, patients discharged home with antibiotics had significantly higher average discharge WBC counts than those discharged without antibiotics (10.6 vs. 9.0, $p<0.001)$. Discharge WBC counts were not compared against postoperative abscess formation since the majority of abscesses developed while patients were still admitted.
A multivariable logistic regression analysis was conducted to determine the impact of exposures of interest on the formation of postoperative abscesses. Of note, initial broad-spectrum antibiotic use was associated with increased odds of postoperative abscess formation $[\mathrm{OR}=3.17,95 \%$ CI $(1.58,6.38)]$. Odds of postoperative abscess formation were also significantly higher among male patients $[\mathrm{OR}=$ $2.18,(1.03,4.60)]$, but were not significant for age, race, or admission WBC count.

\section{DISCUSSION}

Although the utility of intra-abdominal cultures in the setting of perforated appendicitis remains a subject for debate, our analysis shows that the yield of cultures diminishes as the collection moves further away from initial presentation, consistent with previous reports [17]. This is further illustrated by the seven cases where patients initially had bacterial growth on intraoperative culture only to later have a sterile culture at time of postoperative abscess formation. Although all cultures were pre-treated, since antibiotics are initiated prior to appendectomy or radiologic drainage, this trend is likely a product of ongoing antibiotic therapy. When bacterial growth was demonstrated, our 
Table 2 Culture growth by culture type

\begin{tabular}{|c|c|c|c|c|}
\hline & Pre-op drain & Intra-op & Post-op abscess & Blood \\
\hline Total collected (\% w/growth) & $26(96)$ & $46(87)$ & $49(51)$ & $60(5)$ \\
\hline \multicolumn{5}{|c|}{ Total growth (\% by culture type) } \\
\hline Bacteroides fragilis & $23(88)$ & $30(65)$ & $10(20)$ & $1(2)$ \\
\hline Bacteroides vulgatus & - & - & $1(2)$ & - \\
\hline Candida albicans & - & $1(2)$ & $1(2)$ & - \\
\hline Clostridium sp. & $1(4)$ & - & $2(4)$ & - \\
\hline Eggerthella lenta & - & - & $2(4)$ & - \\
\hline Eikenella corrodens & $1(4)$ & $1(2)$ & - & - \\
\hline Enterococcus faecalis & $1(4)$ & - & $2(4)$ & - \\
\hline Enterococcus faecium & - & - & $3(6)$ & - \\
\hline Enterococcus gallinarum & - & - & $1(2)$ & - \\
\hline Escherichia coli & $19(73)$ & $32(70)$ & $10(20)$ & $2(3)$ \\
\hline Fusobacterium sp. & - & $1(2)$ & - & - \\
\hline Gemella sp. & - & $1(2)$ & - & - \\
\hline Granulicatella sp. & - & $2(4)$ & - & - \\
\hline Haemophilus influenzae & - & $1(2)$ & - & - \\
\hline Klebsiella oxytoca & - & - & $1(2)$ & - \\
\hline Klebsiella pneumoniae & - & $2(4)$ & $1(2)$ & - \\
\hline Lactobacillus sp. & - & - & $1(2)$ & - \\
\hline Mixed aerobes & $17(65)$ & $19(41)$ & $4(8)$ & - \\
\hline Mixed anaerobes & $19(73)$ & $18(39)$ & $3(6)$ & - \\
\hline Parvimonas sp. & $2(8)$ & $1(2)$ & - & - \\
\hline Proprionibacterium acnes & - & - & $1(2)$ & - \\
\hline Pseudomonas aeruginosa & $1(4)$ & $7(15)$ & $6(12)$ & - \\
\hline Slackia exigua & $1(4)$ & - & - & - \\
\hline Streptococcus pyogenes & - & $1(2)$ & - & - \\
\hline Viridans group strep & $17(65)$ & $21(46)$ & $10(20)$ & - \\
\hline
\end{tabular}

findings were consistent with the literature, showing B. fragilis, E. coli, and Viridans group streptococcus as the most prevalent organisms $[17,18]$. The rate of $P$. aeruginosa growth has been documented between 17 and 28\% among intraoperative cultures $[4,17,18]$. However, lower rates of $P$. aeruginosa were seen in our cohort: $15 \%$ of all intraoperative cultures and $12 \%$ of all postoperative abscess cultures. This difference may be due to geographic and environmental variability, but is also only an estimate of the true prevalence based on the sample 
Table 3 Sequential surgical site culture summary

Summary of results for patients with both an intra- and postoperative abscess culture collection

\begin{tabular}{lc}
\hline Patients with sequential cultures & 11 \\
Positive intraoperative cultures & 11 \\
Positive postoperative abscess cultures & 4 \\
Sterile postoperative abscess cultures & 7 \\
Identical growth on paired cultures & 2 \\
\hline
\end{tabular}

size of cultures obtained within the cohort. Tailoring antibiotic coverage based on intraoperative cultures may not have an impact on the development of post-surgical complications, although disagreement exists on the need to provide treatment for $P$. aeruginosa $[18,19]$. In our cohort, the majority of patients did not receive cultures and had favorable clinical courses without pseudomonal coverage.

While the use of anti-pseudomonals, specifically, is beyond the scope of this article, our study is not the first to report the overall use of
Table 5 Correlation of average WBC with postoperative abscess formation and/or readmission

\begin{tabular}{lrr}
\hline & & $\boldsymbol{p}$ value \\
\hline Average admission WBC & & \\
Subsequent absess $(n=49)$ & 17.5 & 0.60 \\
No abscess $(n=268)$ & 17.0 & \\
Average discharge WBC & & \\
Subsequent readmission $(n=23)$ & 11.4 & $<\mathbf{0 . 0 1}$ \\
No readmission $(n=309)$ & 9.9 & \\
\hline
\end{tabular}

broad-spectrum antibiotics is associated with worse clinical outcomes [6, 20]. Consideration should be given that patients presenting with a higher level of acuity are more likely to receive broader spectrum antibiotics, accounting for some of this association. In our study, clinical outcomes were considered based on the initial antibiotic regimen, which was decided prior to surgical intervention. While it could not be determined why specific antibiotic regimens were chosen, among patients who received

Table 4 Antibiotic regimen vs. outcomes of interest

\begin{tabular}{|c|c|c|c|c|c|c|c|}
\hline & \multicolumn{2}{|c|}{$\begin{array}{l}\text { Initial antibiotic } \\
\text { treatment }\end{array}$} & \multicolumn{2}{|c|}{$\begin{array}{l}\text { Discharge with } \\
\text { antibiotics }\end{array}$} & \multicolumn{3}{|c|}{$\begin{array}{l}\text { Discharge antibiotics by } \\
\text { route }\end{array}$} \\
\hline & Broad & Narrow & $\overline{\text { Yes }}$ & No & $\overline{\text { PO }}$ & IV & Mixed \\
\hline Average LOS, days & 7.0 & 5.7 & 6.4 & 5.6 & 5.7 & 8.5 & 9.7 \\
\hline$p$ value & $<0.01$ & & 0.02 & 0.13 & & & \\
\hline Average antibiotic duration, days & 13.5 & 10.4 & 13.1 & 6.3 & 11.9 & 16.1 & 18.7 \\
\hline$p$ value & $<0.01$ & & $<0.01$ & $<0.01$ & & & \\
\hline Post-op abscesses (\%) & $25(25)$ & $27(12)$ & $38(22)$ & $14(9)$ & $13(10)$ & $15(71)$ & $10(59)$ \\
\hline$p$ value & $<0.01$ & & $<0.01$ & 0.79 & & & \\
\hline Readmits (\%) & $8(10)$ & $15(6)$ & $14(8)$ & $9(6)$ & $11(8)$ & $1(5)$ & $2(13)$ \\
\hline$p$ value & 0.61 & & 0.37 & 0.39 & & & \\
\hline
\end{tabular}


Table 6 Correlation of admission WBC with key clinical outcomes

\begin{tabular}{|c|c|c|c|c|}
\hline & Patients & $<12$ years & Patients & $>12$ years \\
\hline Median age & 8.4 & & 13.8 & \\
\hline \multicolumn{5}{|l|}{ Total } \\
\hline WBC $0-15$ & 64 & & 46 & \\
\hline WBC $15+$ & 133 & & 74 & \\
\hline \multicolumn{5}{|l|}{ Average LOS } \\
\hline WBC $0-15$ & 5.9 & $p=0.27$ & 6.2 & $p=0.31$ \\
\hline WBC $15+$ & 5.5 & & 6.9 & \\
\hline \multicolumn{5}{|c|}{ Average antibiotic duration } \\
\hline WBC $0-15$ & 9.5 & $p=0.93$ & 11.2 & $p=0.32$ \\
\hline WBC $15+$ & 9.6 & & 12.5 & \\
\hline \multicolumn{5}{|c|}{ Post-op abscesses } \\
\hline WBC $0-15$ & $9(14)$ & $p=0.49$ & $7(15)$ & $p=0.16$ \\
\hline WBC $15+$ & $14(11)$ & & $19(26)$ & \\
\hline \multicolumn{5}{|l|}{ Readmissions } \\
\hline WBC $0-15$ & $4(6 \%)$ & $p=0.95$ & $2(4 \%)$ & $p=0.18$ \\
\hline WBC $15+$ & $8(6 \%)$ & & $8(11 \%)$ & \\
\hline
\end{tabular}

broad-spectrum antibiotics there was a significantly higher rate of postoperative abscess formation, which could indicate a higher level of acuity. However, the length of stay, although significant, is only slightly longer than for patients who received metronidazole and a third-generation cephalosporin $(7.0$ vs. 5.7 days, $p<0.01)$. Additionally, the lack of significant difference in readmission rates between the two groups further suggests that initial management with metronidazole and a third-generation cephalosporin is appropriate and sufficient for most pediatric patients presenting with perforated appendicitis.

Previous studies have shown post-discharge antibiotics lack beneficial value, although this has only been considered in the setting of a normal WBC count at time of discharge [10-13]. Separately, it has also been shown that patients discharged with IV antibiotics experience equal or greater rates of complications than those discharged with oral antibiotics [8, 9]. In our study, patients discharged with PO antibiotics had a statistically significant but clinically insignificant higher average WBC count than patients discharged without antibiotics (9.8 vs. 9.0, $p=0.03)$. In an attempt to account for illness severity, we compared LOS among these groups, an imperfect indicator for acuity, and found no significant difference. Additionally, the lack of difference in postoperative abscess formation and readmission rates supports the practice of discontinuing antibiotics at time of discharge for pediatric patients who are clinically well.

As aforementioned, WBC counts have historically been monitored throughout an admission for perforated appendicitis [10, 21]. In assessing admission WBC count versus other key metrics of interest, we found no significant correlation to suggest there is value in the ability to predict severity of illness. Conversely, discharge WBC counts have historically been an aide in determining whether or not to lengthen an admission or continue antibiotics at time of discharge $[10,11]$. Although a previous study showed that postoperative abscesses are associated with higher rates of persistent leukocytosis 5-7 days postoperatively, no cases of abscess formation occurred among patients tolerating a regular diet 3 days postoperatively [14]. Our analysis showed a significant difference in discharge WBC count among patients who were later readmitted (11.4 vs. $9.9, p<0.01)$, but this difference is not of clinical relevance and would not provide prospective value for the clinician. Thus, our study supports other findings that pediatric patients can be discharged safely when clinically well and without checking a final WBC count [15].

\section{Limitations}

This study is limited as a retrospective, singlecenter analysis and could be improved through subsequent prospective investigation. Similar investigations at multiple centers could help with generalizability, particularly in terms of the microbiology findings. During the period of 
investigation (i.e., 2015-2019), the hospital underwent a transition in electronic medical records, which made certain facets of chart review difficult. Most significantly, a number of patients prior to this transition had documented discharge with antibiotics; however, no duration was listed, and prescribing information was unavailable. To prevent confounding, discharge antibiotics were primarily considered as a binary variable during data analysis, with patients either receiving a prescription or not. While total antibiotic course durations were reported, the actual difference would have been even greater had these data been available.

While the 5-year span of this study provides a robust cohort size, the temporal span may have confounding effects due to changes in antibiotic choices over time. Over the course of the study period, a significant decrease in the use of broad-spectrum antibiotic coverage occurred. Of the 73 patients initially treated with broad-spectrum antibiotics, 44 occurred in 2015, 12 occurred in 2016, and 5 to 6 per year occurred between 2017 and 2019. It is unlikely that this temporal aspect is significantly impacting the final results, since our conclusions generally show no significant difference between those who received broad-spectrum antibiotics and those who did not. It is also possible that initial broad-spectrum antibiotic use reflects illness severity, with sicker patients receiving broader coverage. However the yearover-year trend suggests that initial antibiotic choice may be impacted more by changing provider practice tendencies than by initial patient presentations.

As a single-site study, bacterial growth patterns may reflect geographical influences. The study also included a number of patients who were initially seen at an outside hospital ED and subsequently transferred for escalation of care after the diagnosis of appendicitis was made. In rare cases, it was not always possible to ascertain what interventions were provided at the outside hospital, namely antibiotic administration; however, patients were excluded if a significant portion of their care occurred at another hospital, so the impact on the included patients was likely minimal.

\section{CONCLUSIONS}

This study represents one of the largest and most recent epidemiologic evaluations of perforated appendicitis in children. Importantly, we identified common pathogens of perforated appendicitis, including $B$. fragilis and E. coli, and found that the yield of cultures diminishes over time in the postoperative period. While our analysis found a significant correlation between broad-spectrum antibiotics and postoperative abscesses, the routine use of broad-spectrum antibiotics, or continuing oral antibiotics beyond discharge, was not associated with improved clinically meaningful outcomes over using metronidazole and a third-generation cephalosporin alone until point of discharge. Finally, we found no association between trending WBC counts and clinical outcomes among pediatric patients with perforated appendicitis. Taken together, the findings in this study suggest that pediatric patients presenting with perforated appendicitis may be overtreated, in terms of antibiotic coverage and duration, and the monitoring of WBC counts, beyond the initial diagnosis, is not a reliable tool for this population.

\section{ACKNOWLEDGEMENTS}

Funding. No funding or sponsorship was received for this study or publication of this article.

Authorship. All named authors meet the International Committee of Medical Journal Editors (ICMJE) criteria for authorship for this article, take responsibility for the integrity of the work as a whole, and have given their approval for this version to be published.

Author Contributions. Drs. Plattner, Shakhsheer, and Newland conceptualized and designed the study. Dr. Plattner created the database, conducted data collection and initial analysis, and drafted the initial manuscript. Drs. Shakhsheer and Newland supervised data 
collection. Dr. Wallendorf carried out confirmatory data analysis. All authors critically reviewed the manuscript for important intellectual content.

Disclosures. Alex S. Plattner, Jason G. Newland, Michael J. Wallendorf, and Baddr A. Shakhsheer declare that they have no conflict of interest.

Compliance with Ethics Guidelines. This study received approval from the Washington University in St. Louis institutional review board (IRB), ID \#201908150.

Data Availability. The datasets generated during and/or analyzed during the current study are available from the corresponding author on reasonable request.

Open Access. This article is licensed under a Creative Commons Attribution-NonCommercial 4.0 International License, which permits any non-commercial use, sharing, adaptation, distribution and reproduction in any medium or format, as long as you give appropriate credit to the original author(s) and the source, provide a link to the Creative Commons licence, and indicate if changes were made. The images or other third party material in this article are included in the article's Creative Commons licence, unless indicated otherwise in a credit line to the material. If material is not included in the article's Creative Commons licence and your intended use is not permitted by statutory regulation or exceeds the permitted use, you will need to obtain permission directly from the copyright holder. To view a copy of this licence, visit http://creativecommons.org/licenses/bync/4.0/.

\section{REFERENCES}

1. Stpeter SD. Appendicitis. In: Holcomb GW, Murphy JP, editors. Ashcraft's pediatric surgery. 5th ed. Philadelphia: Saunders Elsevier; 2010. p. 549.

2. Andersen BR, Kallehave FL, Andersen HK. Antibiotics versus placebo for prevention of postoperative infection after appendicectomy. Cochrane Database Syst Rev. 2005;(3).

3. Akingboye AA, Davies B, Tien T. Pus samples in complicated appendicitis: an important investigation or a waste of resources: a prospective cohort study. Scand J Surg. 2019;108(1):55-60.

4. Turel O, Mirapoglu SL, Yuksel M, Ceylan A, Gultepe BS. Perforated appendicitis in children: antimicrobial susceptibility and antimicrobial stewardship. J Glob Antimicrob Resist. 2019;1(16):159-61.

5. Roque FM, Melo Filho AA, Roque AJ, Roque HC, Moreira TM, Chaves EM. Antibiotics for appendicectomy in children and adolescents during the perioperative period: an integrative review. Revista Paulista de Pediatria. 2019;37(4):494-502.

6. Peter SD, Little DC, Calkins CM, Murphy JP, Andrews WS, Holcomb GW III, Sharp RJ, Snyder CL, Ostlie DJ. A simple and more cost-effective antibiotic regimen for perforated appendicitis. J Pediatr Surg. 2006;41(5):1020-4.

7. Arnold MR, Wormer BA, Kao AM, Klima DA, Colavita PD, Cosper GH, Heniford BT, Schulman AM. Home intravenous versus oral antibiotics following appendectomy for perforated appendicitis in children: a randomized controlled trial. Pediatr Surg Int. 2018;34(12):1257-68.

8. Rangel SJ, Anderson BR, Srivastava R, Shah SS, Ishimine P, Srinivasan M, Bryan M, Gong W, Hall M, Localio $\mathrm{R}$, Luan $\mathrm{X}$. Intravenous versus oral antibiotics for the prevention of treatment failure in children with complicated appendicitis: has the abandonment of peripherally inserted catheters been justified? Ann Surg. 2017;266(2):361-8.

9. Acker SN, Hurst AL, Bensard DD, Schubert A, Dewberry L, Gonzales D, Parker SK, Tong S, Partrick DA. Pediatric appendicitis and need for antibiotics at time of discharge: does route of administration matter? J Pediatr Surg. 2016;51(7):1170-3.

10. Desai AA, Alemayehu H, Holcomb GW III, Peter SD. Safety of a new protocol decreasing antibiotic utilization after laparoscopic appendectomy for perforated appendicitis in children: a prospective observational study. J Pediatr Surg. 2015;50(6): 912-4.

11. Bonasso PC, Dassinger MS, Wyrick DL, Smith SD, Burford JM. Evaluation of white blood cell count at time of discharge is associated with limited oral antibiotic therapy in children with complicated appendicitis. Am J Surg. 2019;217(6):1099-101.

12. Fraser JD, Aguayo P, Leys CM, Keckler SJ, Newland JG, Sharp SW, Murphy JP, Snyder CL, Sharp RJ, Andrews WS, Holcomb GW III. A complete course 
of intravenous antibiotics vs a combination of intravenous and oral antibiotics for perforated appendicitis in children: a prospective, randomized trial. J Pediatr Surg. 2010;45(6):1198-202.

13. Gordon AJ, Choi JH, Ginsburg H, Kuenzler K, Fisher J, Tomita S. Oral antibiotics and abscess formation after appendectomy for perforated appendicitis in children. J Surg Res. 2020;1(256):56-60.

14. Dickinson CM, Coppersmith NA, Luks FI. Early predictors of abscess development after perforated pediatric appendicitis. Surg Infect. 2017;18(8): 886-9.

15. Cunningham ME, Zhu H, Hoch CT, DeMello AS, Gusman ND, Fallon SC, Lopez ME. Effectiveness of a clinical pathway for pediatric complex appendicitis based on antibiotic stewardship principles. J Pediatr Surg. 2020;55(6):1026-31.

16. Fallon SC, Kim ME, Hallmark CA, Carpenter JL, Eldin KW, Lopez ME, Wesson DE, Brandt ML, Rodriguez JR. Correlating surgical and pathological diagnoses in pediatric appendicitis. J Pediatr Surg. 2015;50(4):638-41.

17. Dahlberg M, Almström M, Wester T, Svensson JF. Intraoperative cultures during appendectomy in children are poor predictors of pathogens and resistance patterns in cultures from postoperative abscesses. Pediatr Surg Int. 2019;35(3):341-6.

18. Andrey V, Crisinel PA, Prod'hom G, Croxatto A, Joseph JM. Impact of co-amoxicillin-resistant Escherichia coli and Pseudomonas aeruginosa on the rate of infectious complications in paediatric complicated appendicitis. Swiss Med Wkly. 2019;149: 1718.

19. Hamdy RF, Handy LK, Spyridakis E, Dona D, Bryan M, Collins JL, Gerber JS. Comparative effectiveness of ceftriaxone plus metronidazole versus antipseudomonal antibiotics for perforated appendicitis in children. Surg Infect. 2019;20(5):399-405.

20. Kronman MP, Oron AP, Ross RK, Hersh AL, Newland JG, Goldin A, Rangel SJ, Weissman SJ, Zerr DM, Gerber JS. Extended-versus narrower-spectrum antibiotics for appendicitis. Pediatrics. 2016;138(1): e20154547.

21. Anandalwar SP, Graham DA, Kashtan MA, HillsDunlap JL, Rangel SJ. Influence of oral antibiotics following discharge on organ space infections in children with complicated appendicitis. Ann Surg. 2021;273(4):821-5. 